\title{
STATE ESTIMATION FOR TRACKING OF TAGGED SHARKS WITH AN AUV
}

\author{
A Thesis \\ Presented to \\ the Faculty of California Polytechnic State University \\ San Luis Obispo
}

\author{
In Partial Fulfillment \\ of the Requirements for the Degree \\ Master of Science in Computer Science
}

by

Christina Forney

December 2011 
(C) 2011

Christina Forney

ALL RIGHTS RESERVED 


\section{COMMITTEE MEMBERSHIP}

TITLE:

State Estimation for Tracking of Tagged

Sharks with an AUV

AUTHOR:

Christina Forney

DATE SUBMITTED: December 2011

COMMITTEe CHAIR: Chris Clark, Ph.D.

COMMITTEE MEMBER: Zoë Wood, Ph.D.

COMmitTeE MEMBER: Franz Kurfess, Ph.D. 


\begin{abstract}
State Estimation for Tracking of Tagged Sharks with an AUV
\end{abstract}

Christina Forney

Presented is a method for estimating the planar position, velocity, and orientation states of a tagged shark. The method is designed for implementation on an Autonomous Underwater Vehicle (AUV) equipped with a stereo-hydrophone and receiver system that detects acoustic signals transmitted by a tag. The particular hydrophone system used here provides a measurement of relative bearing angle to the tag, but does not provide the sign (+ or -) of the bearing angle. A particle filter was used for fusing measurements over time to produce a state estimate of the tag location. The particle filter combined with an active control system allowed the system to overcome the ambiguity in the sign of the bearing angle. This state estimator was validated by tracking a stationary tag and moving tag with known positions. These experiments revealed state estimate errors were on par with those obtained by manually driven boat based tracking systems, the current method used for tracking fish and sharks over long distances. Final experiments involved the catching, releasing, and an autonomous AUV tracking of a 1 meter leopard shark (Triakis semifasciata) in SeaPlane Lagoon, Los Angeles, California. 


\section{Acknowledgements}

The authors gratefully acknowledge the contribution of National Science Foundation for funding this project. 


\section{Contents}

List of Tables viii

List of Figures $\quad$ ix

1 Introduction 1

2 Related Work 4

2.1 Shark Behavior .................... 4

2.2 Acoustic Tagging and Fish Tracking . . . . . . . . . . . . . . 6

2.3 Robot Tracking . . . . . . . . . . . . . . . . . . 7

2.3.1 Visual Real-Time Tracking of Moving Targets . . . . . . . 7

2.3.2 Tracking with AUVs .............. 8

2.4 State Estimation . . . . . . . . . . . . . . . . . . 9

3 Problem Definition $\quad 11$

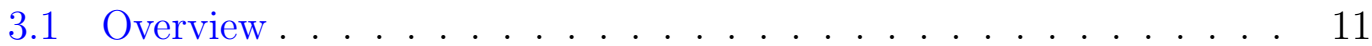

3.2 Estimation Requirements . . . . . . . . . . . . . . . . . 13

4 State Estimator $\quad 16$

4.1 Prediction Step . . . . . . . . . . . . . . . . . 17

4.2 Correction Step . . . . . . . . . . . . . . . . . . . . . . . . . . 19

4.3 Sensor Modeling. . . . . . . . . . . . . . . . . . . . . 21

5 Experiment Description $\quad 22$

5.1 Avila Beach Pier Experiments . . . . . . . . . . . . . . . 23

5.1.1 Stationary Tag . . . . . . . . . . . . . 24

5.1.2 Tracking a Tagged Kayak ............... . 24 
5.1 .3 Tracking a Tagged AUV . . . . . . . . . . . . . 25

5.2 Long Beach Experiments . . . . . . . . . . . . . . . . 26

5.2 .1 Stationary $\operatorname{Tag} \ldots \ldots \ldots \ldots \ldots \ldots$

5.2 .2 Tracking a Tagged AUV . . . . . . . . . . . . . 27

5.2.3 Tracking a Tagged Leopard Shark . . . . . . . . . . . . . 27

6 Results 29

6.1 Stationary $\operatorname{Tag} \ldots \ldots \ldots \ldots$

6.2 Tracking a Tagged AUV . . . . . . . . . . . . . . . . 31

6.3 Tracking a Leopard Shark . . . . . . . . . . . . . . . . . 34

6.4 Particle Convergence Time Series . . . . . . . . . . . . . 35

7 Conclusions 38

8 Future Works 40

9 Appendix 42

$\begin{array}{ll}\text { Bibliography } & 45\end{array}$ 


\section{List of Tables}

4.1 Standard Deviation Values . . . . . . . . . . . . . . . . . . 21

6.1 Comparing error with standard deviation . . . . . . . . . . . 32

9.1 The effects of the number of particles on error . . . . . . . . . 43

9.2 Mission Data . . . . . . . . . . . . . . . . . . . 44 


\section{List of Figures}

3.1 Block Diagram . . . . . . . . . . . . . . . . . . . . 12

3.2 Close-up of Hydrophone Hull Attachment . . . . . . . . . . . 13

3.3 Top Down View of Sample Measurement . . . . . . . . . . . . . 14

4.1 Angle Best Fit Curve . . . . . . . . . . . . . . . . . . 20

5.1 AUV with PVC Rig attached . . . . . . . . . . . . . 22

5.2 AUV with Carbon Fiber Rig attached . . . . . . . . . . . . . 23

5.3 Cal Poly Center for Coastal Marine Science, Avila Beach, CA . . 23

5.4 Stationary Tag Counterweight Buoy . . . . . . . . . . . . . . . 24

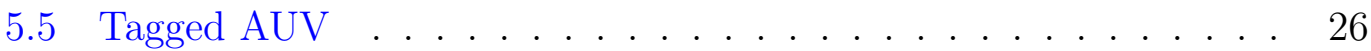

5.6 Seaplane Lagoon, Port of Long Beach, CA . . . . . . . . . . 27

5.7 Leopard Shark . . . . . . . . . . . . . . . . . . . . . . . 28

6.1 Error, Standard Deviation, and Lotek Signal Rate from Tracking a Stationary $\operatorname{Tag} \ldots \ldots \ldots \ldots$. . . . . . . . . . . . 31

6.2 Error, Standard Deviation, and Lotek Signal Rate from Tracking a Tagged AUV . . . . . . . . . . . . . . . . 31

6.3 Trajectories of the tracking AUV-1, the tagged AUV-2, and the Estimated AUV-2 Position are shown . . . . . . . . . . . . . 33

6.4 Close-up of AUV-1, AUV-2, and Estimated Position Trajectories . 33

6.5 Error (m) vs. Signal Rate . . . . . . . . . . . . . . . . . 34

6.6 Standard Deviation and Lotek Signal Rate of Tracking a Tagged Leopard Shark . . . . . . . . . . . . . . . . . . . . . 35

6.7 Trajectories of AUV-1 and Estimated Shark Position _. . . . . 36 
6.8 Time Series of Particle Convergence . . . . . . . . . . . . . . . . . 37 


\section{Chapter 1}

\section{Introduction}

The ocean remains one of the world's most largely unexplored regions. Thus, there is little known about many species that reside in the ocean. Though sharks have been widely researched, there is much to be discovered about shark behavior and movement patterns. In order to increase this knowledge, an autonomous mobile tracking system has been created which will provide researchers with the long term data that has been missing.

Current methods for tracking sharks include remote sensing GPS tags, manual active tracking, and stationary receivers (passive tracking). GPS tags provide accurate positional data, however, these data can only be transmitted when the shark is at the surface [20]. This leaves a gap in information on the location of the shark while not at the surface. Researchers can actively follow sharks with a boat using a mounted receiver; however, this requires human operation to navigate the boat to maintain a signal reading of the tag, and the position of the shark [16] so tracks are limited on temporal scales of hours to days. Finally, stationary acoustic receivers can gather data on the movement of sharks in a localized area. However, these are cumbersome to set up, and when the sharks move out of 
the range of the stationary receivers, data can no longer be recorded. Groups of acoustic receivers can be organized so there are many receivers spread over a specified area, either in high concentration smaller areas, or wide-spread with receivers set up as gates to track the inward and outward movement of sharks and other tagged animals [8]. Unfortunately, none of these solutions address the problem of obtaining high spatial resolution positions on highly mobile species that may easily swim beyond the reaches of a stationary acoustic receiver.

As of yet, there has not been another method deployed to have Autonomous Underwater Vehicles (AUV) track the movement of sharks. In [6] the necessity for en-route decision making in AUVs was was identified as a problem that needed to be addressed. AUVs have been programmed to follow a designated GPS waypoint path, recording information as it travels. Prior to this project, there had yet to be an AUV that could continually follow a single tag on a specific animal (shark) and make logical decisions on the changing location to follow the animal. An active localization of the shark is necessary to track and follow it as it moves. A major part of this active localization is the sensor fusion required for such state estimation. The AUV was equipped with a stereo-hydrophone receiver system which provided differential time of arrival data necessary for state estimation. This paper presents a Particle Filter based method for fusing measurements from the stereo-hydrophone receiver over time, enabling real-time estimation of the shark state.

This paper is organized as follows. Section II discusses related works and elaborates on existing research. The problem definition is described in Section III. Section IV describes the state estimator, and breaks down the steps of the proposed algorithm. Experiments are described in Section V, and Section VI reports the results of these experiments. Section VII concludes the scientific 
contributions made by this project. Finally, Section VIII discusses future work to be done to further advance research in this area. 


\section{Chapter 2}

\section{Related Work}

This project requires an integration of knowledge and technology from various disciplines including biology, robotics, and computer science. Tracking of aquatic animals has been greatly improved through the use of GPS. Additionally, the field of robotics, specifically underwater robotics, has been improved through the incorporation of new sensors and actuators used on robots. A significant contribution has been made with the integration of these two fields. The merging of this information is the basis of this project. The following sections discuss the current technologies and knowledge available for these two disciplines, including shark behavior, the tagging and tracking of sharks, tracking using robots, and state estimation algorithms.

\subsection{Shark Behavior}

A goal of this research project is to determine fine scale behavioral patterns of a variety of shark species. To initiate this project the research team will focus on tracking Leopard sharks and White sharks. An important consideration when 
tracking sharks is the ability of the AUV to keep up with shark swimming speeds. It was found that the top swimming speeds of white sharks is $4.8 \mathrm{~km} / \mathrm{hr}$, with an average sustainable traveling speed of $3.2 \mathrm{~km} / \mathrm{hr}$. Leopard sharks are a much smaller species which travel with an average speed of $1.94 \mathrm{~km} / \mathrm{hr}$. White sharks can travel up to $190 \mathrm{~km}$ within a three to four day time period [2], whereas leopard sharks tend to stick to smaller areas, such as within bays. When hunting, White sharks become more aggressive in their swimming patterns, they circle more frequently, and have higher swimming speeds. In contrast, leopard sharks are predators of opportunity; they will wait patiently until something comes within their range [16].

Behaviors of sharks differ with age. Adult sharks tend to move in long linear lines following coasts or traveling between islands $[1,11]$. Juvenile sharks tend to stay in bays or surrounding islands rather than traveling far distances. Adult sharks are typically solitary creatures, only interacting with other sharks during mating. In contrast, Juveniles stay in small groups, and appear much more social.

When using AUVs in close proximity of sharks, there is a concern with respect to the sensitivity to electromagnetic fields. All sharks have a sense called electroreception, though White sharks are especially sensitive [15]. Electroreception is a shark's primary means of navigation, and is also used during hunting. The AUV emits an electromagnetic field which could potentially attract the attention of a shark, causing them to come investigate the robot. A shark's investigation of a foreign object typically involves trying to eat the object. This could potentially pose a problem for the robot.

There is still much unknown about the behaviors of sharks. No data has been collected on the fine-tuned swimming patterns, the mating patterns, or the lifespan of these sharks. Due to the current available tagging and tracking devices 
it has been impossible to gather this data. Long term (weeks to months) data on shark movement is non-existent. This project aims to address this issue by creating a prototype mobile tracking system that can follow the shark for long periods of time across large distances.

\subsection{Acoustic Tagging and Fish Tracking}

Current tracking of aquatic animals includes stationary receivers, receivers on boats, and GPS tags. Stationary receivers can track tag information while multiple tagged individuals are within range. However, once the animal leaves the area where the receivers are positioned, no data can be gathered. This is problematic for both stationary locations near the coast, as well as out in the ocean $[8]$.

GPS tags provide a longer term solution, as they provide data consistently and are not restricted to a single area. However, once the shark dives below the surface of the water, the GPS signal can no longer be detected [20]. Ship-bourne receivers and directional hydrophones have been used by humans to steer the boat with the goal of following the tagged shark. However, boats can disturb the sharks and potentially change the behavior of the shark. In addition, this requires the human to maintain operation of the vessel and follow the signal of the shark for the length of the track.

In [22] stationary receivers were set up in a bay, and data was gathered on a tagged Shovelnose shark which came into range. This particular study aims to validate the use of acoustic tags and hydrophone receivers. Additionally, from the data that was gathered, the researchers were able to characterize multiple shark behavior states based on the shark's motion patterns. They were thus able 
to create a shark behavior state estimator to run on their acquired data.

The stereo-hydrophone receiver and acoustic tag that will be used during this study will provide measurements to the state estimator, including bearing to the tag, and signal strength. This data is used to calculate a velocity, orientation, and position estimate of the shark relative to the AUV.

\subsection{Robot Tracking}

Robotics is a highly idolized field, and is thus a heavy area of research. Humans desire to have tasks automated for them, which explains the push in industry for robotic intelligence. Particle filter estimation has long been used in robot intelligence, and has greatly improved the performance of automated robots. AUV's have been used to follow GPS waypoint paths through the ocean, however intelligent online decision making based on the results of an "unpredictable" animal is a new and exciting endeavor. In the following sections, robot systems that perform tracking, multiple object tracking, and underwater data acquisition are discussed.

\subsubsection{Visual Real-Time Tracking of Moving Targets}

To interact safely and efficiently with dynamic environments, robots must be able to track and follow moving objects. For example, there have been several projects developed to accomplish dynamic tracking systems based on vision. In [13], joint probabilistic data association filters are used in conjunction with particle filters in order to track multiple humans inside a building, and are able to successfully and reliably keep track of multiple persons [13]. The joint prob- 
abilistic data association filter is an algorithm that improves the separation and individual identification of data when tracking multiple objects. This particular study compares the success of Kalman filters to the success of particle filters when tracking a moving being. They found that they were restricted when using Kalman filters due to the Gaussian distributions over the estimated state. Kalman and particle filters are algorithms which create estimates of state, either in the object being tracked, or in localization of the robot itself. A Gaussian distribution is used by the filters in order to predict the state of the object or robot. An additional study, [14], also used particle filters and joint probabilistic data association filters to determine location of people in an office type environment.

Similarly, in [19] visual data is acquired by the robot in order to determine it's desired movement path. That particular study focused on soccer playing robots which need to track the location of a soccer ball in-order to determine their next move. In [13], a particle filter algorithm is used to predict the location of the ball. Another related work, [21] uses a SLAM (simultaneous localization and mapping) algorithm in order to determine the surroundings. This particular study determines that the computationally intensive algorithm is infeasible to be used in real time. Thus, they implemented a simpler SLAM algorithm which was able to successfully track moving objects from a moving vehicle in a crowded urban area.

\subsubsection{Tracking with AUVs}

Underwater robots have also been equipped with vision systems to track moving objects [5]. While in [23] a vision system was developed to conduct tracking of fish with an ROV, it was not implemented for autonomous tracking experiments. 
In [12], a vision system was used to successfully track jelly-fish with an ROV. AUVs have been equipped with acoustic receivers to passively record acoustic tag signals. In [7] an AUV was used to gather data from two tagged Atlantic Sturgeon in the Hudson River. This study proved that AUV's are highly useful in gathering data on a tagged fish. The AUV was sent on a mission to perform sweeping trajectories across the designated area where the tagged Sturgeon were located. As the robot was close to the tag signal, it picked up the Sturgeon's location. This is a related study, researchers chose not to follow the Sturgeon, and rather, gather general data about the fishes' location in the area.

In [6] scientists aimed to validate introducing hydrophone receivers onto an AUV. They were able to validate the hydrophone's effectiveness in gathering information from tags in the area of deployment. The key discovery in this paper was that signal reception was acquirable, and distance and angle (thus, position) can be determined from the tag signal picked up by the moving hydrophones.

\subsection{State Estimation}

Kalman filter algorithms are often used in estimating state in robot localization problems. Based on a distribution of error, Kalman filters use uni-modal Gaussian distributions for representations of state. Kalman filters are very efficient when used for localization[18], but due to the limitations of the uni-modal distribution, are best used when the initial position of the robot is known [3].

Another approach to robot localization is Monte Carlo Localization (MCL), a computationally efficient localization algorithm with the ability to represent arbitrary distributions [3]. MCL uses an adaptive sampling mechanism in which the number of sample states is chosen as the robot travels. A larger sample set is 
used when position is relatively unknown, and thus, MCL can globally localize a robot [3]. Particle filter estimation is heavily based on the MCL algorithm [18]. A particle filter state estimation algorithm approximates a belief state through a set of particle representations [17]. Each particle represents a single randomized representation of state, the set of which creates a multiple hypothesis sample set. Each particle is given a probability weighting based on its accuracy to sensor readings. This weighting provides a scheme for re-sampling the particle set, where the particles with higher probabilities are much more likely to be chosen than the particles with low probabilities. Particle filters have been used in robotics to aid in robot navigation and state recovery $[4,9]$. In this paper, the particle filter's ability to handle ambiguous sensor measurements is leveraged to deal with a stereo hydrophone and receiver system that cannot determine the sign of the relative angle to a detected fish tag. 


\section{Chapter 3}

\section{Problem Definition}

The problem that this project aims to solve can be defined as follows. Given an AUV with stereo-hydrophone and receiver, design an estimator that determines the position and velocity of a tagged shark in real time. In order to solve this problem, hardware and software configuration must be considered. The following sections discuss an overview of the system, the challenges associated, and details the components found within.

\subsection{Overview}

AUV Sensor Suite The AUV used in this project is an Oceanserver IVER2 AUV (Fig. 5.2), a torpedo shaped robot actuated with two fins to control pitch, two rear fins to control yaw, and a rear propeller to provide locomotion. The AUV's antenna has a built-in GPS receiver providing longitude and latitude measurements at a rate of $1 \mathrm{~Hz}$. These position measurements are represented here as $Z_{G P S}$. The IVER2 also has a 3 degree of freedom compass. In this work the compass' yaw measurement $Z_{\theta}$ is required for shark state estimation. 


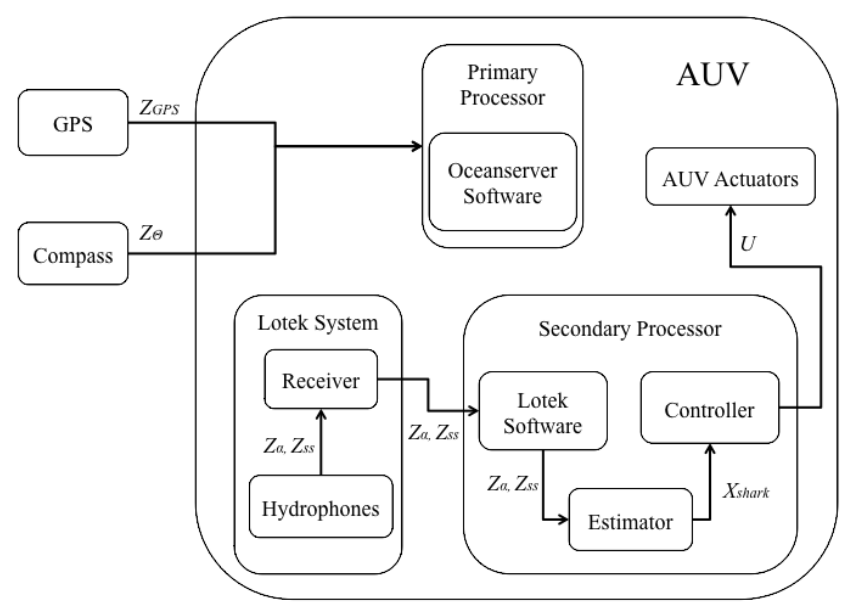

Figure 3.1: Block Diagram

AUV Actuators The AUV actuators are four servo motors to actuate the control surfaces (i.e. fin angles), and one motor to spin the propeller. As shown in Figure 3.1, $U$ represents the control vector sent to each of these five motors.

Primary Processor The primary processor runs waypoint tracking missions, monitors the status of the robot's actuators, and enables sensor and actuator communications. The monitoring is performed by the Oceanserver software which takes $Z_{\theta}$ and $Z_{G P S}$ as inputs from the sensor suite.

Secondary Processor The secondary processor is designated for external programs, and is where the acoustic receiver software, estimator, and controller are run. The receiver software produces measurements of the bearing to the tag $Z_{\alpha}$ and signal strength $Z_{s s}$, and passes these measurements to the estimator. The estimator processes the inputs, and outputs $X_{\text {shark }}$ which it sends to the controller. The controller takes $X_{\text {shark }}$ as an input, and uses this to make decisions about movement of the AUV relative to the estimated shark position. 


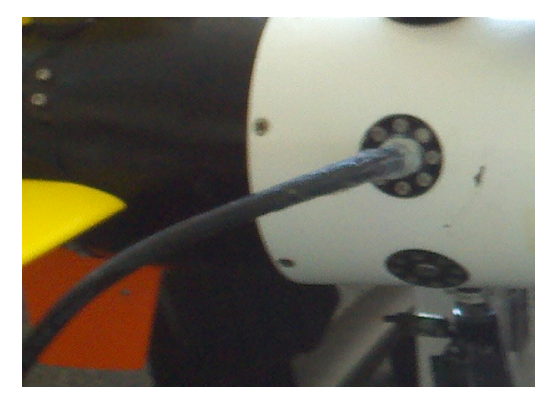

Figure 3.2: Close-up of Hydrophone Hull Attachment

Stereo Hydrophone System The stereo-hydrophones, acoustic receiver, and receiver software are part of the Lotek MAP RT-A Hydrophone sensor system. The AUV has been adapted to internally contain the receiver circuit board from the Lotek MAP RT-A Hydrophone sensor system. The hydrophone system is designed to listen for frequencies centered at $76 \mathrm{kHz}$, the same frequency of signals emitted by the Lotek tags. The tags transmit encoded analog signals that allow them to be identified uniquely on the same frequency. The hydrophone cables are internally connected and fed through sealed holes in the tail end of the hull of the AUV, as seen in Figure 3.2. The external portion of the hydrophone cables are 1.5 meters, and 2 meters in length.

\subsection{Estimation Requirements}

The estimation problem, the core work of this thesis, is depicted in Figure 3.3. In this figure a top down view of this system is shown with hydrophones $h 1$ and $h 2$ positioned just ahead of the AUV nose and just behind the AUV tail, respectively. $X_{\text {auv }}$ represents the position and yaw of the AUV with respect to an inertial coordinate frame and determined by OceanServer's proprietary software. The estimator uses $X_{a u v}$ and $Z_{\alpha}$ as inputs to estimate the shark position and velocity $X_{\text {shark }}$ at each time step $t$. More precisely, for $t \in\left[0, t_{\text {max }}\right]$ : 


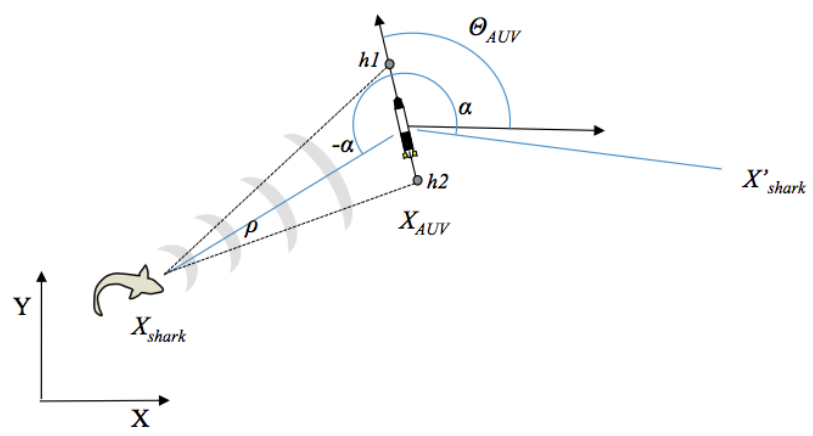

Figure 3.3: Top Down View of Sample Measurement

Given:

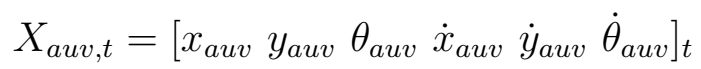

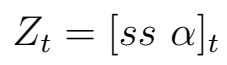

Determine:

$$
X_{\text {shark }, t}=\left[\begin{array}{llll}
x_{\text {shark }} & y_{\text {shark }} & \theta_{\text {shark }} & v_{\text {shark }} \\
w_{\text {shark }}
\end{array}\right]_{t}
$$

Such that:

$$
\begin{gathered}
-8 \leq \alpha \leq 8 \\
0 \leq s s \leq \max S S \\
L=\rho_{\max }
\end{gathered}
$$

Challenges associated with the stereo-hydrophone system include its limited range $(L=100 \mathrm{~m})$, its low resolution $(=\pi / 9 \mathrm{rad})$, and the ambiguity of sign of the bearing angle. This ambiguity is illustrated in Figure 3.3, where the AUV 
cannot determine if a single bearing measurement $\alpha$ corresponds to angle $+\alpha$ or $-\alpha . X_{\text {shark }}^{\prime}$ represents the other possible location of state based on the ambiguous sensor reading. Signal strength, ss, represents a Lotek measurement of approximate distance, power level, and interference. It gives a general representation of how far away the tag is, however in this project, this value was not used for state estimation. The Lotek input value of angle, $\alpha$, as mentioned above, is an integer value, and represents the angle between the AUV and the tag as seen in Figure 3.3. The values which represent state of the shark and AUV are the $\mathrm{x}$ and $\mathrm{y}$-coordinate frame values, the rotation $\theta$, and the previous time step's values of that state designated with a dot, e.g. $\dot{\theta}_{\text {shark }}$. Each of these states is defined for a given time step $t$. The maximum range of the Lotek system is designated $L$, and represents the greatest distance that the Lotek system can pick up a signal.

The estimator is used in two ways, in real-time tracking on board the AUV, and offline to improve the state estimate accuracy. Real-time estimation is used during the physical tracking of a tag. The estimator outputs $X_{\text {shark }}$ to the controller, which makes movement decisions based on that state. Offline, the estimator is used with both simulated and real AUV/acoustic sensor data. When using the estimator with real sensor data, the accuracy of the filter can be improved by tuning paramaters of the filter. If position data of the tag is known, optimal filter paramaters maybe determined offline and implemented later for real-time state estimation. 


\section{Chapter 4}

\section{State Estimator}

A Particle Filter (PF) was used to estimate the state of the shark, with states defined in equation 3.3. The PF uses a collection of $P$ particles to represent a probabilistic distribution of potential shark states. Each particle represents a single estimate of the shark state, with a position, orientation, velocity, and weight. Initially, each particle is randomly assigned to a position, orientation, and velocity, by selecting from a uniform random distribution. Positions $(x, y)$ are randomly selected from an $L$ meter by $L$ meter square area with the initial location of the AUV as the center of the distribution. Here, $L$ reflects the range of the acoustic receiver system.

$$
\begin{gathered}
x_{\text {shark }}^{p} \in\left[x_{\text {auv }}-L / 2, x_{\text {auv }}+L / 2\right] \\
y_{\text {shark }}^{p} \in\left[y_{\text {auv }}-L / 2, y_{\text {auv }}+L / 2\right] \\
\theta_{\text {shark }}^{p} \in[-\pi, \pi]
\end{gathered}
$$




$$
v_{\text {shark }}^{p} \in\left[0, v_{\max }\right]
$$

After the distribution of particles is initialized and randomly distributed, particles are updated with the $\mathrm{PF}$ algorithm that is called at each iteration of the AUV's control loop. The algorithm has two main steps, a preditction step and a correction step. The prediction step predicts the shark state of every particle. If a new valid signal from the shark tag is received, the likelihood or weight of all particles is calculated and the correction step will be called to resample the particle distribution. At the end of these two steps, the shark state estimate is calculated as the average position, orientation and velocity of all $P$ particles.

\subsection{Prediction Step}

At every time step, each of the $P$ particles in the set $\left\{X^{p}\right\}$ is propagated forward according to a first-order motion model. The motion model is a function of the previous particle position $\left(x_{\text {shark }}^{p}, y_{\text {shark }}^{p}\right)$, orientation $\theta_{\text {shark }}^{p}$, velocity $v_{\text {shark }}^{p}$ and the uncertainty associated with these values, specifically the standard deviations $\sigma_{\theta}$ and $\sigma_{v}$. Steps $3-8$ in Algorithm 1 show details. Randomness is added to each propagated state by sampling from a Gaussian distribution with zero mean and standard deviations $\sigma_{\theta}$ and $\sigma_{v}$ (i.e. with the function $\operatorname{randn}()$ in Algorithm 1).

The new position is determined on lines 5 and 6 , and is calculated by adding the previous value with a velocity and direction computation relative to the amount of time that has passed, $\Delta t$. The velocity is based on the previous velocity and the velocity after the prediction, so, to further filter velocity, it is 


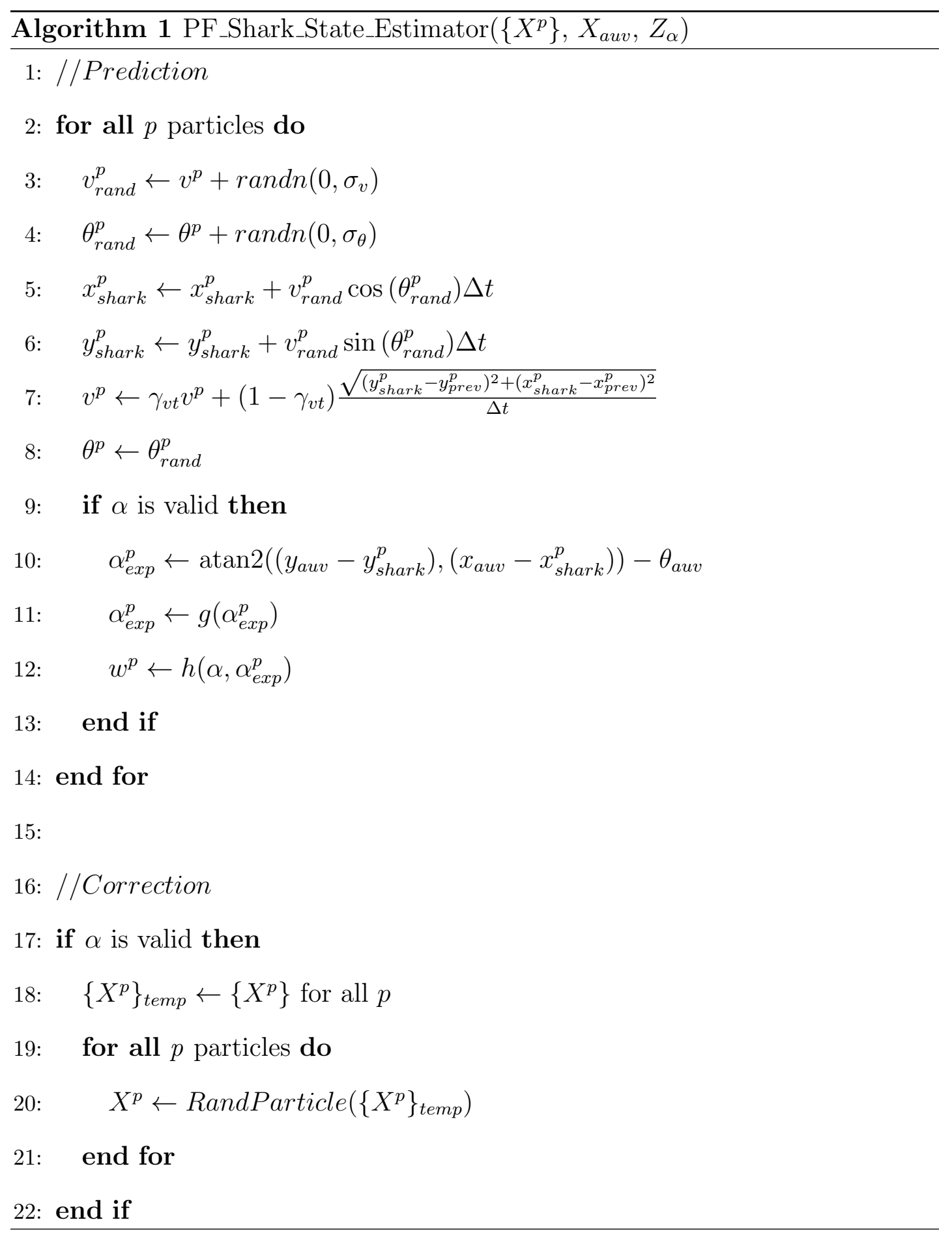


calculated using a weighted average of current estimate with the previous estimate. A weighting value of $\gamma_{v t}$ is used to determine the dependency on new versus previous estimates within the average.

This prediction step allows the PF to maintain an estimate on the position of the shark, even when the shark is moving. The prediction causes the particles to spread outward during times when the signal from the acoustic tag is weak or blocked. The particles are propagated the approximate velocity of a shark so that the distribution area covers the entire area that the shark could have traveled in that period of time.

\subsection{Correction Step}

This correction step is defined in Algorithm 1 lines 10 - 12. The correction and re-sampling are only run when a "valid" Lotek value is received. The expected bearing angle form the AUV to the particle's shark position, $\alpha_{e x p}^{p}$ is calculated on line 10, and is adjusted for the rotation of the AUV, $\theta_{\text {auv }}$, (see Figure 3.3). On line 11, the angle $\alpha_{e s t}^{p}$ is then converted from units of radians to a Lotek angle units with the following function:

$$
\begin{aligned}
g\left(\alpha_{\text {exp }}^{p}\right)= & -1 * 10^{-6}\left(\alpha_{\text {exp }}^{p}\right)^{3}+2 * 10^{-5}\left(\alpha_{\text {exp }}^{p}\right)^{2} \\
& +0.0947 \alpha_{\text {exp }}^{p}-0.2757
\end{aligned}
$$

The above function was defined through experimental testing of the Lotek system, and was generated from a Least Squares best fit line to those data plots. See Figure 4.1 for an example unit conversion. The angle, $\alpha_{e x p}^{p}$, is then rounded to the nearest whole number, since all Lotek angle values are integers between - 8 and 8 . 


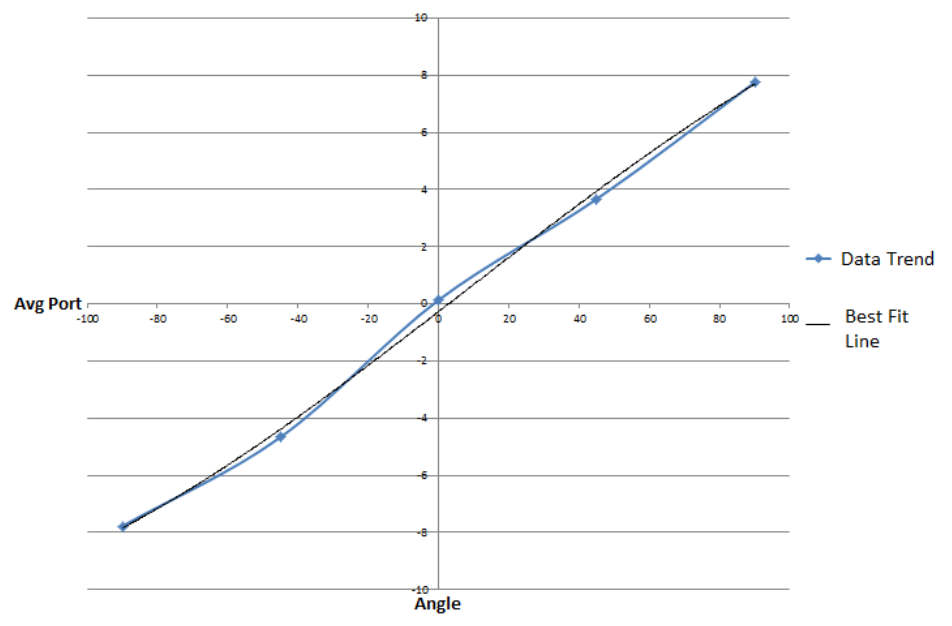

Figure 4.1: Angle Best Fit Curve

The particle is then assigned a weight on line 12, through the following Gaussian weighting function:

$$
h\left(\alpha, \alpha_{e x p}^{p}\right)=0.001+\frac{1}{\sqrt{2 \pi \alpha_{e x p}^{p}}} * e^{\frac{-\left(\alpha_{e x p}^{p}-\alpha\right)^{2}}{2 * \sigma_{a l p h a}^{2}}}
$$

The weight has a minimum value of 0.001 , and is given a higher value when the particle's expected angle, $\alpha_{\text {exp }}^{p}$, is closer to the measured angle, $\alpha$. As the angle difference decreases, a higher weighting is assigned.

The re-sampling is shown in Algorithm 1, Lines 18 - 21. A copy of the propagated particle set is saved in $\left\{X^{p}\right\}_{\text {temp }}$. Then, each particle state is repopulated by randomly selecting from $\left\{X^{p}\right\}_{\text {temp }}$ using the function RandParticle(). This function selects a particle at random, with a likelihood of selection proportional to the particle's normalized weight. To improve the robustness of the algorithm, a small \% of particles returned by this function will be newly generated random states. 


\subsection{Sensor Modeling}

There is a certain amount of error associated with every motion model propagation and sensor measurement. These errors are modeled as random variables that follow a zero mean Gaussian probability density function. The standard deviations associated with these functions were derived both with experimental and historical data. The $\sigma$ values in Table 4.1 represent the standard deviations used within this work. Standard deviation is a measure of the dispersion of a set of data from its mean. For example, the $\sigma$ is chosen based on the accuracy and trust in the variable containing error. The accuracy of the variable is within $\sigma$ units from the saved value of the variable. This means that at any given time, the variable value could be off by $\sigma$ units in any direction. It is important to account for this error within values in order to accurately describe the state.

Table 4.1: Standard Deviation Values

\begin{tabular}{|c|c|}
\hline$\sigma$ Name & Value \\
\hline \hline$\sigma_{\text {auv }}$ & 5.0 meters \\
\hline$\sigma_{v}$ & 0.3 meters per second \\
\hline$\sigma_{\theta}$ & $\pi / 2$ radians \\
\hline$\sigma_{\alpha}$ & 1.0 lotek angle value \\
\hline$\sigma_{s s}$ & 15 lotek signal value \\
\hline$\gamma_{v t}$ & 0.75 \\
\hline
\end{tabular}

A higher $\sigma$ value represents a larger distribution of error, whereas a small value represents a smaller distribution indicating a greater level of trust in the accuracy of that value. 


\section{Chapter 5}

\section{Experiment Description}

Two external frames were created in order to hold the stereo-hydrophones in place. The Lotek MAP RT-A system was designed to have the hydrophones set 2.4 meters apart, and at least one meter below the surface of the water. The first rig is made from PVC, has two support beams which attach to the AUV by hose clamps through a t-bracket shaped PVC attachment, see Figure 5.1. The second rig is made of carbon fiber, and has a similar attachment mechanism to the PVC, however the rig support beams are angled as seen in Figure 5.2.

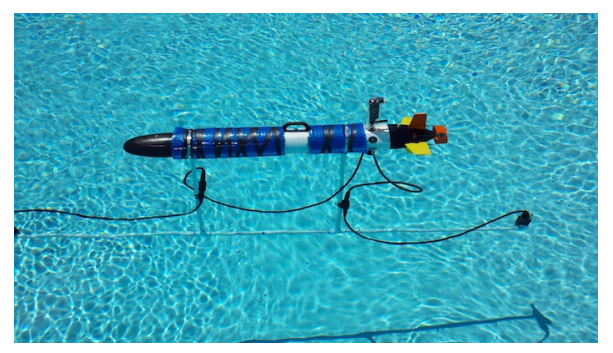

Figure 5.1: AUV with PVC Rig attached

Experiments were performed with both rigs, and are described in the following sections. 


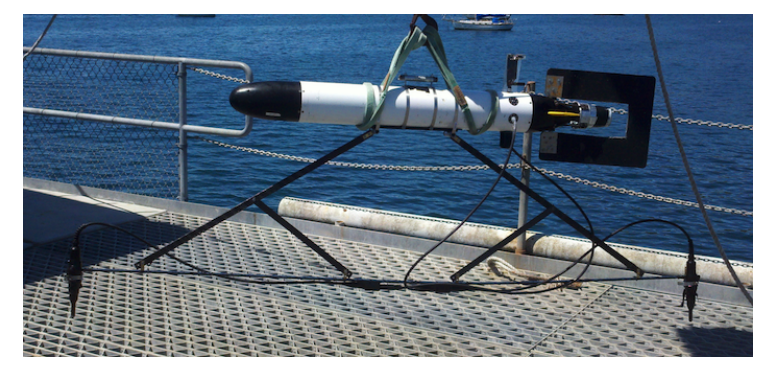

Figure 5.2: AUV with Carbon Fiber Rig attached

\subsection{Avila Beach Pier Experiments}

A series of validation experiments were performed at the Cal Poly Center for Coastal Marine Science (CCMS). The facility is located at the end of a large pier in Avila Beach, CA, as an be seen in Figure 5.3, and is made up of a building for teaching, a filtration management building, a boat locker, and a lowered platform which provides direct access to the ocean below. This platform was used to launch the AUV and Kayak. These experiments included sensor characterization (e.g. determine $\sigma_{\alpha}$ ), AUV tracking of a stationary tag, and AUV tracking of a moving tag.

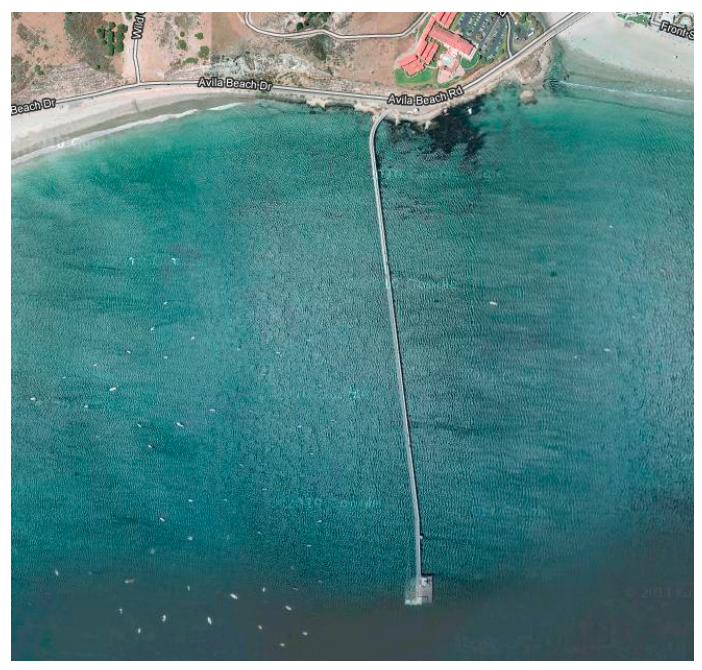

Figure 5.3: Cal Poly Center for Coastal Marine Science, Avila Beach, CA 


\subsubsection{Stationary Tag}

At CCMS, experiments were performed using a stationary tag. The tag was attached to a 10 meter rope approximately two meters below the surface of the water. The water depth in the Avila Bay testing area was approximately 10.0 meters deep. Figure 5.4 shows how two large shackles were tied to one end, anchoring the tag in a stationary position. To counteract the movement of the waves and the tides, the rope was fed through a buoy and then a counterweight of another smaller shackle was tied to the other end of the rope. During experiments, the AUV's start position relative to the tag was varied to ensure tracking could be performed from every direction. In addition to the multiple starting locations, starting points were tested at different initial distances from the tag. AUV start positions also were varied according to initial distance to the tag (i.e. 20, 50, 75, and 100 meters). For stationary tag experiments, the AUV-1 terminated its mission when it was within 10 meters of the tag.

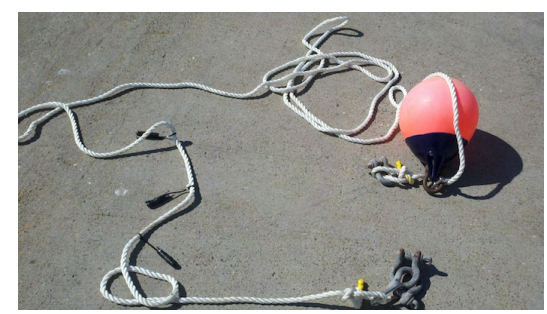

Figure 5.4: Stationary Tag Counterweight Buoy

\subsubsection{Tracking a Tagged Kayak}

A three meter long rope was tied to the stern (back end) of a kayak. A small shackle was tied to the loose end of the rope to submerge the tag. The acoustic tag was attached to the rope approximately 2 meters below the surface of the water, which was a depth of 9 meters. Once the tag was set up and the kayak was 
in the water, the kayak was set in a semi-stationary location for the AUV to track. Semi-stationary refers to the drifting movement of the kayak which occurs in the ocean and the presence of wind. A slowly moving kayak was also tracked with AUV starting locations within each quadrant surrounding. Starting distances of $25 \mathrm{~m}, 50 \mathrm{~m}, 75 \mathrm{~m}$, and $100 \mathrm{~m}$ were used. During travel, the moving kayak traveled in a linear fashion parallel to AUV, directly towards the AUV, perpendicular to the AUV, and directly away from the AUV. These different travel lines helped verify multiple starting orientations.

\subsubsection{Tracking a Tagged AUV}

The same three meter rope used while tracking a tagged kayak was attached to a $6^{1} /{ }_{4}$ inch stainless steel hose clamp, which was secured around the nose of the second AUV (AUV-2). A small shackle was tied to the trailing end of the rope to help submerge the tagas seen in Figure 5.5. Once fully configured, the tag was approximately two meters below the surface. GPS measurements were recorded at the surface just above the tag's location. The depth of the water in Avila Bay is approximately 10.0 meters. This experiment refers to two different AUVs, so, the AUV performing the tracking with the acoustic hydrophone system will now be referred to as AUV-1. The AUV acting as a shark will be referred to as AUV-2. AUV-2 was manually driven in large circular patterns around AUV-1, with straight lines of movement interspersed.

Once the AUV-1 was deployed for these experiments, it would autonomously track the tag's position estimates produced by the PF. To note, a controller was implemented that would achieve two goals: Minimize the distance between the $A U V$ and tag and Minimize the time in which particles converge to the correct 
position of the tag.

Given the direction to the tag is $\gamma_{t}=\alpha_{t}+\theta_{A U V, t}$, the controller directed the AUV-1 to maintain its maximum propeller speed, while repeating on the following 3 steps: 1) track a desired heading of $\theta_{\text {des }}=\gamma_{t}+\pi / 4$, then 2) track a desired heading of $\theta_{\text {des }}=\gamma_{t}-\pi / 4$, and finally 3) track a desired heading of $\theta_{\text {des }}=\gamma_{t}$. This resulted in the AUV-1 zig-zagging its way towards the AUV-2 with 90 degree turns that help resolve the ambiguity in the sign of the bearing angle.

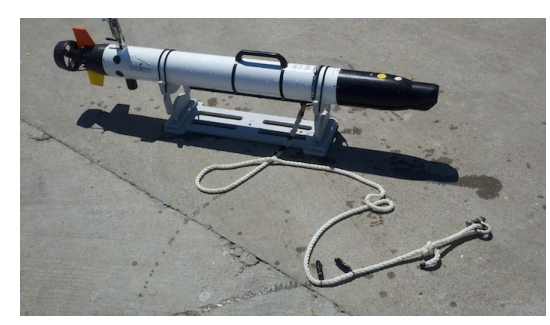

Figure 5.5: Tagged AUV

\section{$5.2 \quad$ Long Beach Experiments}

In Long Beach, CA, as seen in Figure 5.6, the experiments from CCMS were repeated to verify accuracy and functionality at a new location. In addition to these same experiments, a leopard shark (Triakis semifasciata) was caught, externally fitted with an acoustic transmitter, and tracked. The AUV was set up with the carbon fiber rig during the first few days, and on the final two days, while tracking a shark, the PVC rig was used. It was necessary to shorten the $\mathrm{PVC}$ rig to compensate for the shallower waters of the lagoon. In some parts of the lagoon, eel grass became a problem both for AUV navigation and attenuation of the acoustic signal. The PVC support beams were shortened to 0.75 meters in length. 


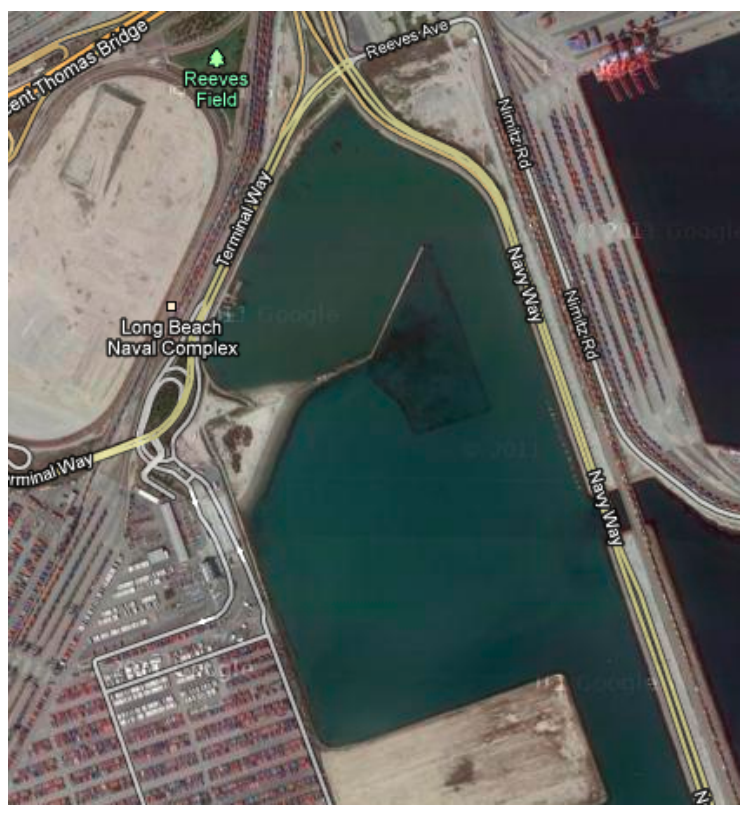

Figure 5.6: Seaplane Lagoon, Port of Long Beach, CA

\subsubsection{Stationary Tag}

Experiments were the same as at the CCMS.

\subsubsection{Tracking a Tagged AUV}

Experiments were the same as at CCMS, however, AUV-2 was driven as though it were a shark skirting around the edges of the lagoon, and was tracked for a much longer time period.

\subsubsection{Tracking a Tagged Leopard Shark}

A Leopard Shark, see Figure 5.7 [10] needed to be caught in order to tag and follow a live shark. To catch a leopard shark for final validation of the system, a 10 hook long line was set in the lagoon and continuously monitored. Although several species of sharks were caught and released, a 1-meter leopard shark was 
externally dart tagged with an acoustic transmitter (Lotek MM Series, $76 \mathrm{kHz}$ freq, 2,5 second ping rate), which is in standard use for tagging large marine fishes. The shark was pulled to the surface using the line, and was then gently restrained with a rope tied to its tail. Sharks have a biological response of tonic immobility, becoming still as though they are hypnotized, when they are turned upside down. This was used to keep the shark from moving while it was fitted with the tag. The entire procedure took less than 10 minutes. Once the tagged shark was released, the AUV was deployed to track and follow the shark.

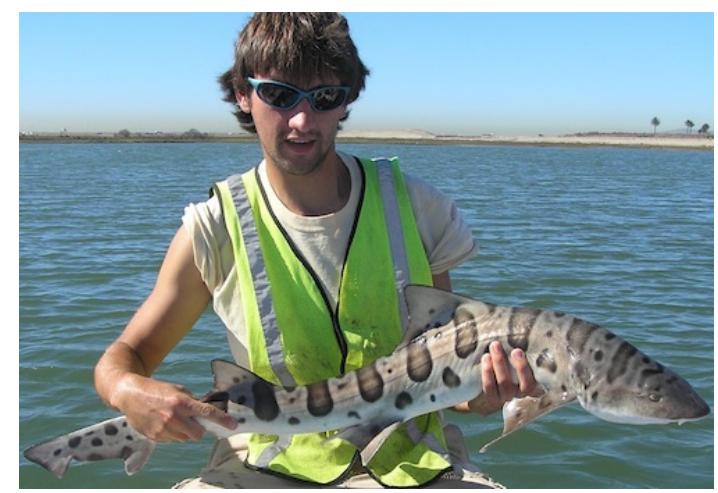

Figure 5.7: Leopard Shark 


\section{Chapter 6}

\section{Results}

A particle filter estimation is used to track the state and location of sharks to better determine and understand their behavior. As demonstrated in [22], particle filter estimation can decrease the error in location predictions of sharks. By combining the use of this estimation algorithm and the separate states of shark behavior characterized by the velocity and distance traveled, the location of the sharks can be closely predicted. This allows the deployment of an AUV, with mounted acoustic tag receivers, to have the ability to follow a shark through the ocean on larger scale and longer term voyages. This will enable acquisition of detailed information regarding the behavior of shark movement. Though currently the AUV running time is limited by battery usage, the algorithm has the capabilities for long term tracking.

In this experiment, acoustic tags are used as in $[8,7,6]$ to determine the location of the shark. The accuracy of the acoustic transmitters on the AUV were tested to determine the weighting and variance properties for weighting calculations. Based on the accuracy of the transmitters, a level of variance has been calculated to estimate the movement of the shark, and corrected based on 
the delayed travel response of the location from the acoustic tag. With a proper behavior state algorithm, in addition to an accurate variance level, the AUV was successfully deployed to follow the sharks.

The following sections describe the results from tracking a stationary tag, tracking a tagged AUV, tracking a tagged Leopard Shark, and shows an example of the particle convergence which occurred during these tracks.

\subsection{Stationary Tag}

A stationary tag was anchored approximately 1.5 meters below the surface at a recorded GPS location. AUV-1 was deployed at multiple locations, and was set to track the stationary tag. The AUV tracked the stationary tag to a location within 10 meters of the tag, then terminate the mission. The error during a typical experiment, as defined in equation 6.1, can be seen plotted in black in Figure 6.1. This error remains less than 18 meters during the experiment, and is on average less than 10 meters. The overall standard deviations, in blue and green represent the spread of the particles a higher standard deviation value equates to a higher uncertainty in estimated location. The overall performance of the algorithm is seen through the average error, maximum error, and minimum error over time. Error is defined as the distance between the actual and estimated tag position:

$$
e_{t}=\sqrt{\left.\left(\hat{x}_{\text {shark }}-x_{\text {shark }}\right)^{2}+\left(\hat{y}_{\text {shark }}-y_{\text {shark }}\right)^{2}\right)}
$$

Signal rate, i.e. the frequency of usable measurements, is also plotted in Figure 6.1, in the color magenta. Notice that as the signal rate gets lower, the 
error rate increases. This correlation is expected because when there are no signals, a greater error and standard deviation in the estimated position of the tag is seen. When the signal rate is higher, there is a corresponding drop in error and standard deviation levels, showing that when more signals are received the AUV is better able to determine an accurate location of the tag.

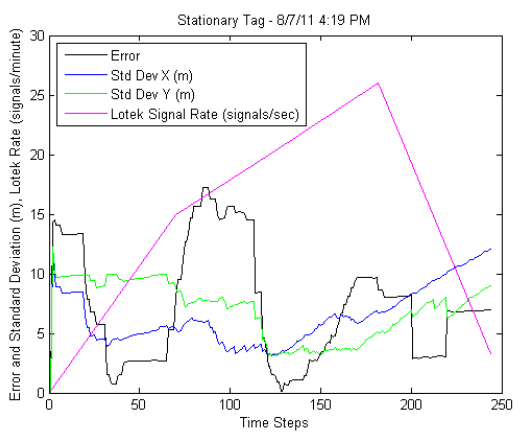

Figure 6.1: Error, Standard Deviation, and Lotek Signal Rate from Tracking a Stationary Tag

\subsection{Tracking a Tagged AUV}

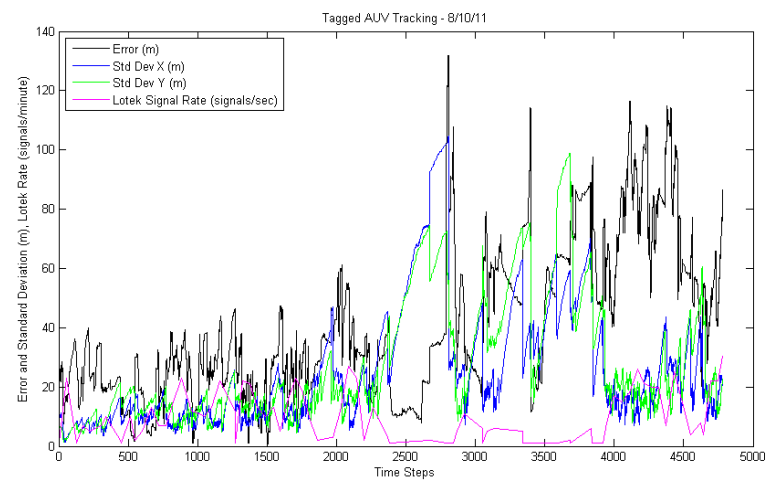

Figure 6.2: Error, Standard Deviation, and Lotek Signal Rate from Tracking a Tagged AUV

To demonstrate system performance with a moving target, results are presented from an experiment where an acoustic tag with a signal rate of 30 sig- 
Table 6.1: Comparing error with standard deviation

\begin{tabular}{|c|c|}
\hline Comparison & Proportion of smaller error \\
\hline$e_{x}<\sigma_{\text {avg } X}$ & $36.22 \%$ \\
\hline$e_{y}<\sigma_{\text {avg } Y}$ & $41.55 \%$ \\
\hline$e_{x}<\sigma_{X}$ & $22.29 \%$ \\
\hline$e_{y}<\sigma_{Y}$ & $25.99 \%$ \\
\hline$e<\sigma$ & $42.12 \%$ \\
\hline
\end{tabular}

nals/minute, was attached to a second Iver2, AUV-2. Fig. 6.3 shows the paths for both the tracking vehicle (named AUV-1) and the tagged vehicle (named AUV-2). AUV-2 was manually driven within the lagoon, mimicking the relatively slow movement of a leopard shark. Figure 6.4 is a close up view of that same track. AUV-2 recorded GPS locations of its path, allowing a comparison of the actual location of the tagged AUV-2, and the estimated position predicted by AUV-1. AUV-1 autonomously tracked and followed AUV-2 using the PF and controller described above. The error, standard deviations, and signal rate can be seen in Figure 6.2. At $\mathrm{t}=2500$ seconds, there is a significant increase in error. This corresponds with poor quality acoustic measurements we observed as the AUVs crossed an area with a high density of eel grass. This can be observed as this darker coloring in Fig. 6.3. As mentioned previously, eel grass creates a curtain of dampening in signal transmission, and as the AUV enters this area, there is a significant drop in signal rate. Figure 6.5 shows error compared to signal rate. The general inverse trend can be seen in the linear best fit line indicated in red. As signal rate increases, error decreases.

The proportion of the time error was less than the standard deviation of the 


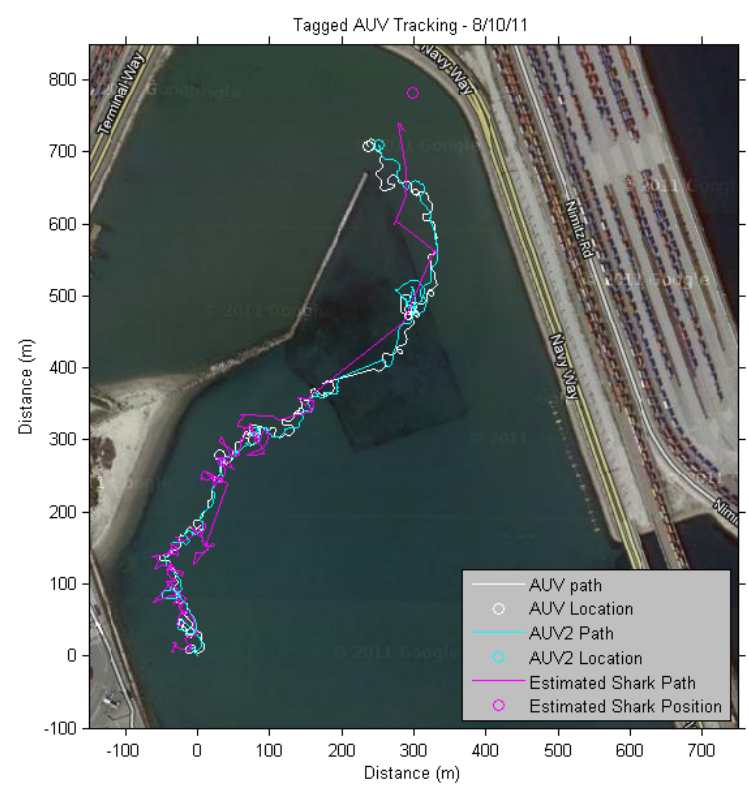

Figure 6.3: Trajectories of the tracking AUV-1, the tagged AUV-2, and the Estimated AUV-2 Position are shown

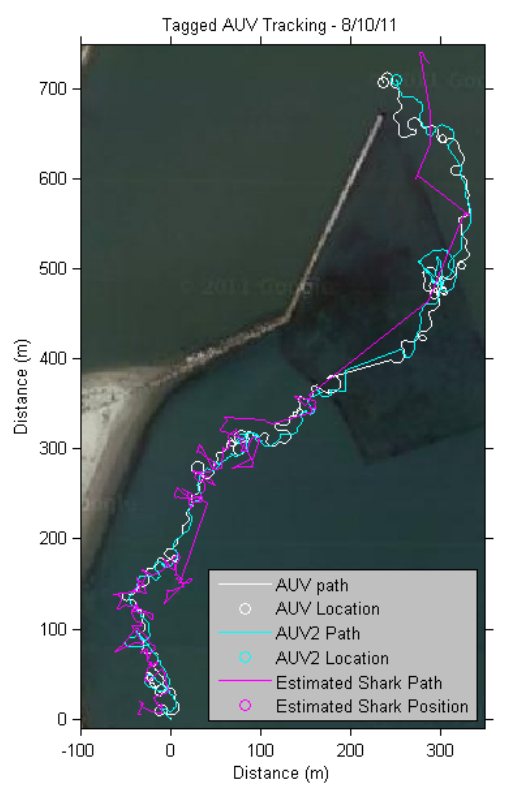

Figure 6.4: Close-up of AUV-1, AUV-2, and Estimated Position Trajectories 


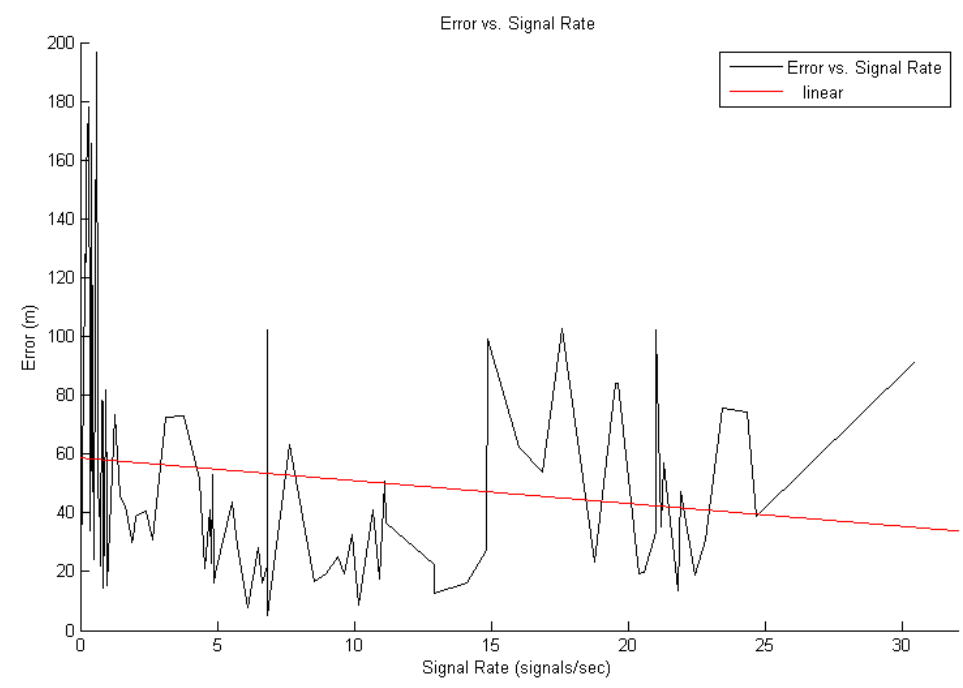

Figure 6.5: Error (m) vs. Signal Rate

particle spread is shown in Table 6.1. Standard deviations are confidence to this effect. Errors were less than the distance error $42.12 \%$ of the time. Distance error is determined with the following equation.

$$
e_{t}=\sqrt{\left(x_{s t d D e v}^{2}+y_{s t d D e v}^{2}\right)}
$$

\subsection{Tracking a Leopard Shark}

On August 9, 2011, a tagged leopard shark was tracked by the AUV for several hours with little interruption. The AUV-1 and estimated shark paths from a 48minute long tracking experiment are shown in $6.7 \mathrm{a}$ and $6.7 \mathrm{~b}$. AUV-1 was deployed at a location where the acoustic signal was picked up by the on-board tracking system. Figure 6.7a shows a close up of the trajectories of AUV-1, as well as the estimated position of the shark when the standard deviation of the particle set was less than or equal to ten meters in both the $\mathrm{X}$ and $\mathrm{Y}$-coordinate plane. 
Figure $6.7 \mathrm{~b}$ is a zoomed-out version of the trajectories, this shows the location within Sea Plane Lagoon that the actual tracking took place. Figure 6.6 shows the corresponding standard deviations of the particle set as well as the signal rate from the acoustic tag. While no estimation accuracy was obtained, these experiments demonstrated the ability for long term autonomous AUV tracking and following of a live shark. Table II summarizes the results, with a notable maximum tracking time of $1.67 \mathrm{hrs}$.

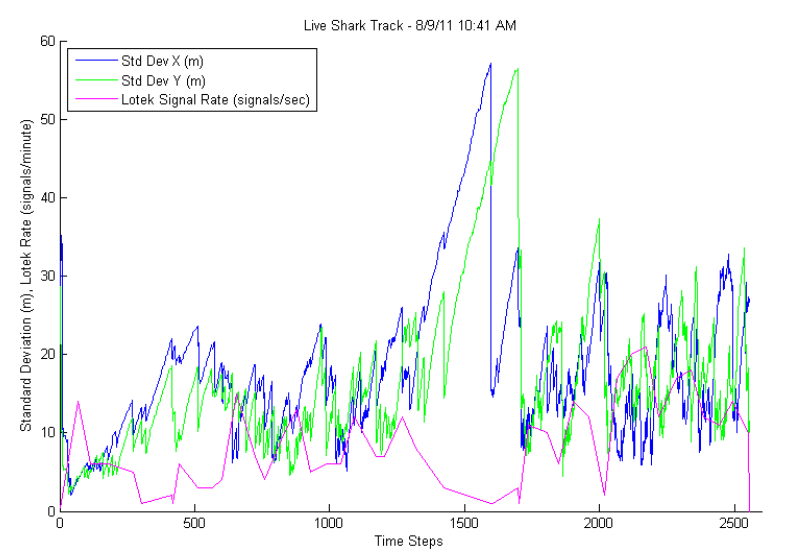

Figure 6.6: Standard Deviation and Lotek Signal Rate of Tracking a Tagged Leopard Shark

\subsection{Particle Convergence Time Series}

In Figure 6.8, a series of images represent the convergence of particles while tracking a tagged shark. In $6.8 \mathrm{a}$, the initial time step, the particles are randomly distributed throughout an $L$ meter by $L$ meter square area centered around the initial location of the AUV. The second image, 6.8b, shows the beginning of particle convergence after a single acoustic signal is picked up by the hydrophones. The ambiguity in the sign of $\alpha$ can be observed here by the fact that particles are into two symetrical groups, one on each side of the AUV. The third image, 


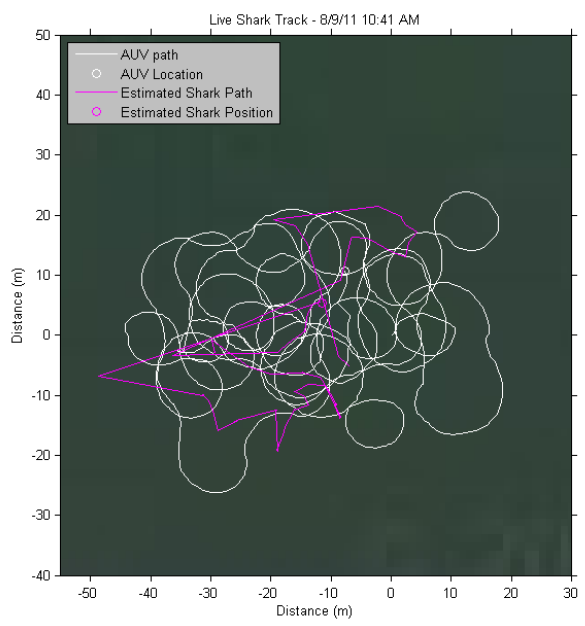

(a) Close-up

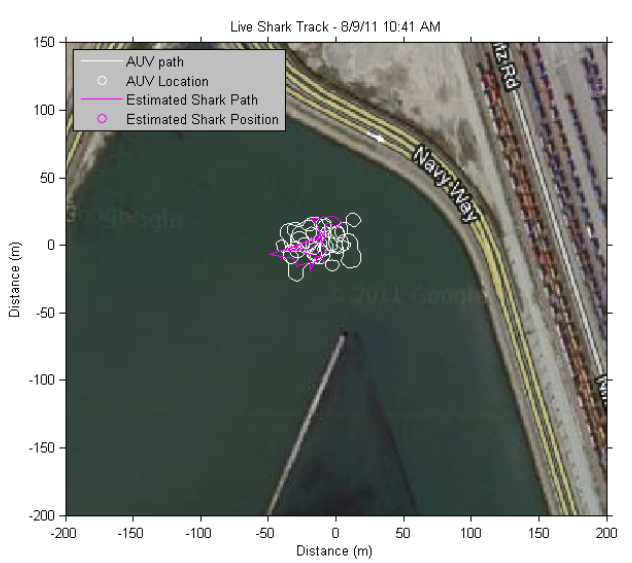

(b) Far View

Figure 6.7: Trajectories of AUV-1 and Estimated Shark Position

6.8c, depicts an instance when the AUV has rotated enough so that only one of the rays cast by the current bearing measurement $\left(+Z_{\alpha}\right.$ or $\left.-Z_{\alpha}\right)$ overlap with one of the existing particle groups. This geometric overlap leads to appropriate weighting of particles and convergence to a single accurate location. After a few more signals from the tag, and only 32 seconds after the initialization, the particles have consolidated into a tight distribution in Table 6.8d.

These four images demonstrate the convergence that occurred during each experiment. The particles continually spread out through propagation, then were weighted and re-sampled after a Lotek measurement was obtained. It was a repeated cycle of expansion and contraction, with frequent contractions during a higher Lotek signal rate.

The optimal number of particles was determined by running experiments with varying numbers of particles, and charting the minimum, maximum, and average error with that number of particles as seen in 9.1. 


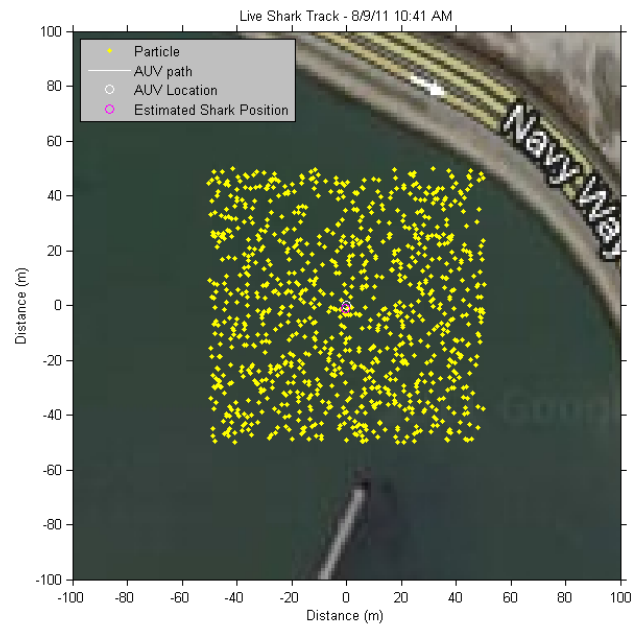

(a) $\mathrm{t}=0.0 \mathrm{~s}$

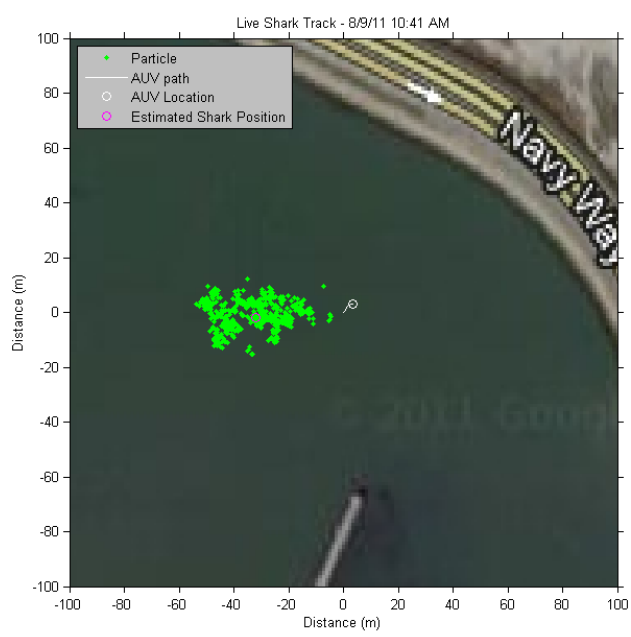

(c) t $=13.92 \mathrm{~s}$

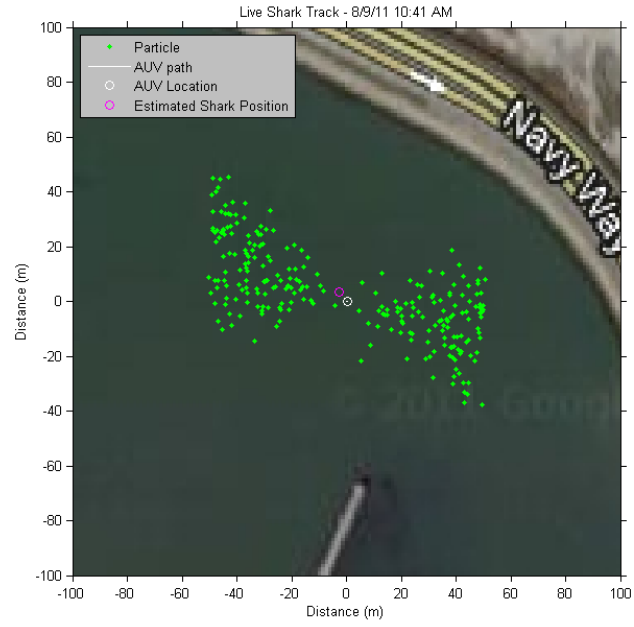

(b) $\mathrm{t}=2.54 \mathrm{~s}$

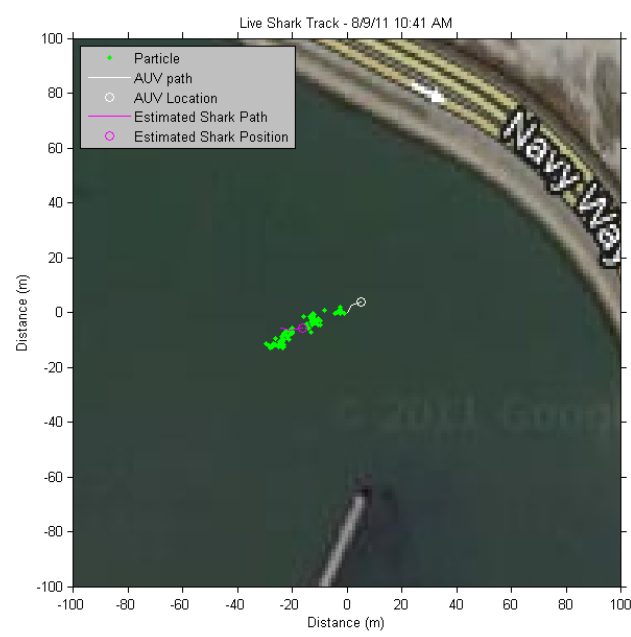

(d) $\mathrm{t}=32.37 \mathrm{~s}$

Figure 6.8: Time Series of Particle Convergence 


\section{Chapter 7}

\section{Conclusions}

A state estimation method has been developed to enable tracking and following of a tagged sharks. There are still improvements to be made, but the existing framework for this project has been established. It is now known that a shark can be autonomously tracked by an AUV. A state estimator is presented such that a position can be maintained on a tagged Leopard shark. The state estimator uses a particle filtering algorithm containing prediction and correction steps which control the movement of the particles. The prediction step moves the particles forward based on a velocity, rotation, and time elapsed. The correction step weights the particles on the accuracy of position based on the input $\alpha$ value from the Lotek hydrophone receiver system. At the end of each correction step, the particles are re-sampled based on their weights. This causes particles that more closely reflect the $\alpha$ value to be represented in higher quantities, while the outliers are likely to disappear. The state estimator is what determines the position of the tag, and is the basis for en-route decisions. This filtering algorithm has been proven accurate through testing by localizing a stationary tag, tracking a tagged AUV, and a tagged shark. While tracking a second tagged AUV, the 
average error during the tracking was 41.73 meters, with a minimum value of 0.85 meters. A shark was continually tracked for a period of 1 hour and 41 minutes, thus validating this real system. The link between biology and robotics is clear; data that could not be collected by biologists previously is now within reach. 


\section{Chapter 8}

\section{Future Works}

Signal strength of the tag should be more carefully calibrated with both an external sensor system as well as with the Lotek system in place. This could provide valuable range measurement, which may be required in tracking faster swimming sharks. It is unclear whether it will be possible to track a larger, and more quickly moving shark with the current system. There is some question whether the AUV will be able to keep up with the pace of these faster swimming sharks, and maintain a location of this shark. A better range in the sensor system will be required in order to maintain a track on these faster swimming sharks. The current system's signal strength was barely adequate for maintaining a location on the very slow swimming Leopard Sharks. With a larger range, the AUV will be able to maintain a track on a shark even when it chooses to leave an area and travel further from the position of the AUV. If the shark chooses to leave at a quick enough pace it will swim out of range of the current system, and unless it chooses to circle back around and re-enter the range area of the AUV, the track will be terminated. With a larger range, the distance that the shark can travel before the track is lost is much greater. In addition to the need for 
a better acoustic system, a longer battery life of the AUV must be realized. At this time the battery of the AUV can support the system for approximately 10 hours of continuous running. With the duration of tracking is currently limited by this battery power.Streamlining and reduction of the hydrophone profile will improve battery life of the AUV, reduce the likelihood of animal disturbance, and make the AUV more manuverable. Finally, this work promotes the use of collaborative multi-AUV tracking that may improve accuracy and reduce the likelihood of losing the shark. 
Chapter 9

Appendix 
Table 9.1: The effects of the number of particles on error

\begin{tabular}{|c||c|c|c|}
\hline Particles & Mean (m) & Max (m) & Min (m) \\
\hline 1000 & 26.29 & 55.67 & 0.910 \\
\hline 500 & 25.85 & 54.27 & 1.949 \\
\hline 400 & 27.99 & 57.03 & 3.50 \\
\hline 300 & 27.30 & 55.19 & 2.28 \\
\hline 200 & 27.98 & 56.72 & 4.13 \\
\hline 100 & 28.72 & 57.80 & 13.06 \\
\hline
\end{tabular}




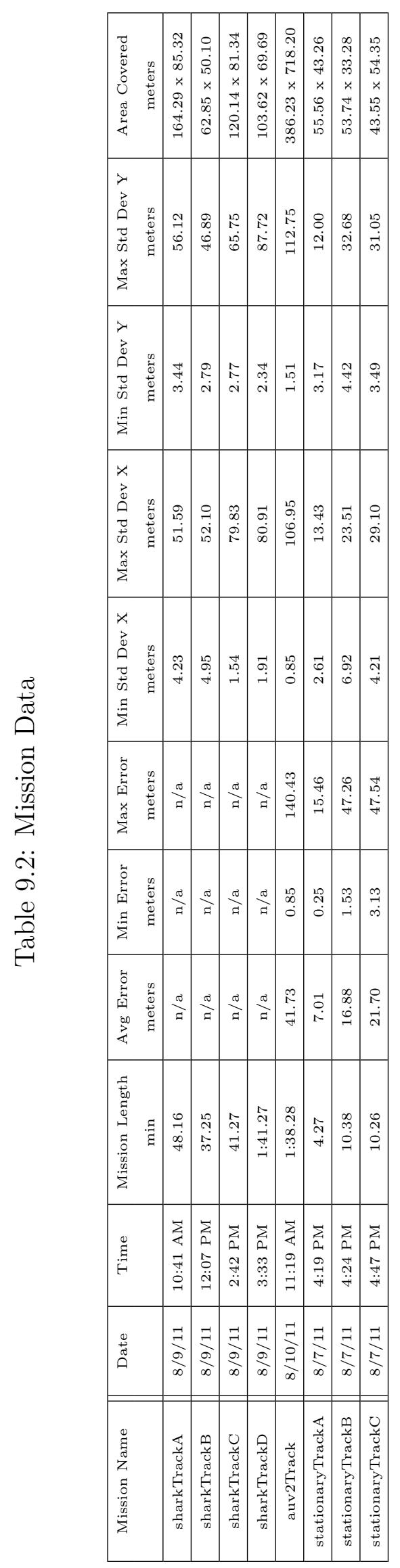




\section{Bibliography}

[1] B. Bruce, J. Stevens, and H. Malcolm. Movements and swimming behaviour of white sharks (carcharodon carcharias) in australian waters. Marine Biology, 150(2):161-172, 2006.

[2] F. Carey, J. Scharold, and A. Kalmijn. Movements of blue sharks (prionace glauca) in depth and course. Marine Biology, 106(3):329-342, 1990.

[3] D. Fox, W. Burgard, F. Dellaert, and S. Thrun. Monte carlo localization: Efficient position estimation for mobile robots. In Proceedings of the National Conference on Artificial Intelligence, pages 343-349. JOHN WILEY \& SONS LTD, 1999.

[4] D. Fox, S. Thrun, W. Burgard, and F. Dellaert. Particle filters for mobile robot localization. 2001.

[5] C. Georgiades, A. German, A. Hogue, H. Liu, C. Prahacs, A. Ripsman, R. Sim, L. Torres, P. Zhang, M. Buehler, et al. Aqua: an aquatic walking robot. In Intelligent Robots and Systems, 2004.(IROS 2004). Proceedings. 2004 IEEE/RSJ International Conference on, volume 4, pages 3525-3531. IEEE, 2004.

[6] T. Grothues and J. Dobarro. Fish telemetry and positioning from an au- 
tonomous underwater vehicle (AUV). Instrumentation ViewPoint, (8):78, 2009.

[7] T. Grothues, J. Dobarro, J. Ladd, A. Higgs, G. Niezgoda, and D. Miller. Use of a multi-sensored AUV to telemeter tagged atlantic sturgeon and map their spawning habitat in the hudson river, USA. In Autonomous Underwater Vehicles, 2008. AUV 2008. IEEE/OES, pages 1-7. IEEE, 2008.

[8] T. M. Grothues. A review of acoustic telemetry technology and a perspective on its diversification relative to coastal tracking arrays. Tagging and Tracking of Marine Animals with Electronic Devices, Diversification of Acoustic Telemetry Technology:77-90, 2009.

[9] F. Gustafsson, F. Gunnarsson, N. Bergman, U. Forssell, J. Jansson, R. Karlsson, and P. Nordlund. Particle filters for positioning, navigation, and tracking. IEEE Transactions on Signal Processing, 50(2):425-437, 2002.

[10] A. Murch. Leopard shark pictures - triakis semifasciata images.

[11] A. Peter Klimley, S. Beavers, T. Curtis, and S. Jorgensen. Movements and swimming behavior of three species of sharks in la jolla canyon, california. Environmental Biology of Fishes, 63(2):117-135, 2002.

[12] J. Rife and S. M. Rock. Segmentation Methods for Visual Tracking of DeepOcean Jellyfish using a Conventional Camera. IEEE Journal of Oceanic Engineering, 28(4):595-608, 2003.

[13] D. Schulz, W. Burgard, D. Fox, and A. Cremers. Tracking multiple moving targets with a mobile robot using particle filters and statistical data association. In Robotics and Automation, 2001. Proceedings 2001 ICRA. IEEE International Conference on, volume 2, pages 1665-1670. IEEE, 2001. 
[14] D. Schulz, W. Burgard, D. Fox, and A. Cremers. People tracking with mobile robots using sample-based joint probabilistic data association filters. The International Journal of Robotics Research, 22(2):99, 2003.

[15] C. Sepulveda, S. Kohin, C. Chan, R. Vetter, and J. Graham. Movement patterns, depth preferences, and stomach temperatures of free-swimming juvenile mako sharks, isurus oxyrinchus, in the southern california bight. Marine Biology, 145(1):191-199, 2004.

[16] D. Sims, E. Southall, A. Richardson, P. Reid, and J. Metcalfe. Seasonal movements and behaviour of basking sharks from archival tagging: no evidence of winter hibernation. Marine Ecology Progress Series, 248:187-196, 2003.

[17] S. Thrun, W. Burgard, and D. Fox. Probabilistic robotics. In Probabilistic Robotics (Intelligent Robotics and Autonomous Agents), USA, sept. 2005. The MIT Press.

[18] S. Thrun, D. Fox, W. Burgard, and F. Dellaert. Robust monte carlo localization for mobile robots. Artificial Intelligence, 128(1-2):99-141, 2001.

[19] A. Treptow and A. Zell. Real-time object tracking for soccer-robots without color information. Robotics and Autonomous Systems, 48(1):41-48, 2004.

[20] F. Voegeli, M. Smale, D. Webber, Y. Andrade, and R. O'Dor. Ultrasonic telemetry, tracking and automated monitoring technology for sharks. Environmental Biology of Fishes, 60(1):267-282, 2001.

[21] C. Wang, C. Thorpe, S. Thrun, M. Hebert, and H. Durrant-Whyte. Simultaneous localization, mapping and moving object tracking. The International Journal of Robotics Research, 26(9):889, 2007. 
[22] A. Xydes, M. Moline, C. Lowe, and C. Clark. Behavior characterization and particle filter localization of a shovelnose shark, 2009.

[23] J. Zhou and C. M. Clark. Autonomous fish tracking by rov using monocular camera. Computer and Robot Vision, Canadian Conference, 0:68, 2006. 